ERRATUM

doi:10.1038/nature15717

\title{
Erratum: Structural imprints in vivo decode RNA regulatory mechanisms
}

Robert C. Spitale, Ryan A. Flynn, Qiangfeng Cliff Zhang,

Pete Crisalli, Byron Lee, Jong-Wha Jung,

Hannes Y. Kuchelmeister, Pedro J. Batista, Eduardo A. Torre, Eric T. Kool \& Howard Y. Chang

Nature 519, 486-490 (2015); doi:10.1038/nature14263

In Fig. $2 \mathrm{a}$ of this Letter, an error in the placement of the labels on the pie chart was introduced during the production process. The correct numbers were reported, but the size of the pie sectors was incorrect. In addition, on page 488, we made an incorrect attribution of the acronym 'Fox'. The Fox family of RBP is named after the gene 'feminizing locus on X' (PMID: 7821230), rather than 'forkhead box'. We apologize for this oversight. These corrections do not affect the conclusions of the paper, and both errors have now been corrected in the online versions of the paper. 